\title{
Sepsis and septic shock: a practical management accordling to Sepsis-3 diagmostic criteria
}

\author{
Matteo Giorgi-Pierfranceschi \\ Emergency Department, Hospital of Val d'Arda, AUSL Piacenza, Italy
}

\section{Introduction}

In the last two decades, the management of sepsis became out of Intensive Care Unit (ICU). ${ }^{1}$ Most of the affected patients are admitted to internal medicine wards, thus internists need to have some simple, quick and repeatable tools to get diagnosis and to promptly initiate the adequate therapy and monitor the goals.

\section{Diagnosis of sepsis}

The reliable identification of patients suffering from sepsis and septic shock is not simple because there is no gold standard diagnostic test. ${ }^{2}$ Indeed, the diagnosis of sepsis requires clinicians to interpret a number of nonspecific physiological and laboratory abnormal findings among patients with suspected or definite infection. ${ }^{3,4}$ Moreover, to make the diagnosis of severe sepsis, clinicians need to understand whether a patient has an infection and an acute or chronic organ dysfunction, and if the two events are related. All these evaluations can be subjective and clinicians might differ in their judgments. As a consequence of this background, there is a significant variability in how clinicians diagnose sepsis and this inhomogeneity has important im-

Correspondence: Matteo Giorgi-Pierfranceschi, Emergency Department, Hospital of Val d'Arda, AUSL Piacenza, via Roma 35, 29017 Fiorenzuola d'Arda (PC), Italy. Tel.: +39.0523.989605.

E-mail: m.giorgi@ausl.pc.it

Key words: Sepsis; septic shock; Sepsis-3 diagnostic criteria.

Received for publication: 12 October 2016.

Accepted for publication: 20 October 2016.

This work is licensed under a Creative Commons Attribution NonCommercial 4.0 License (CC BY-NC 4.0).

CCopyright M. Giorgi-Pierfranceschi, 2016

Licensee PAGEPress, Italy

Italian Journal of Medicine 2016; 10:376-377

doi:10.4081/itjm.2016.803 plications for clinical care, epidemiologic and clinical studies, public health surveillance, pay-for-performance initiatives, and quality improvement programs. ${ }^{5}$

In last February 2016 the Third International Consensus Definitions for Sepsis and Septic Shock (Sepsis-3) has been published, in that consensus paper, sepsis was defined by the authors as life-threatening organ dysfunction caused by a deregulated host response to infection. A fundamental step in the new definition for sepsis and septic shock is still the demonstration of an underlying infection, for which we refer to other chapters in this monograph, but we strongly support the use of focused ultrasonography. According to this formulation sepsis is defined as evidence of infectious disease accompanied by organ dysfunction, considering that even a low degree of organ dysfunction related to an infectious disease (suspected or confirmed) can deteriorate further, and needs a prompt and appropriate diagnosis and intervention (antimicrobials and supportive cares) because it is associated with an higher in-hospital mortality. Certainly, nonspecific SIRS criteria such as fever, hypothermia, tachycardia, or change in white blood cells count will continue to help in diagnosing infections, but they are extremely nonspecific and widespread in hospitalized patients both related and not related to infections. Moreover, using these new criteria in clinical practice, the definition severe sepsis becomes superfluous because even patients presenting with modest dysfunction can worsen, emphasizing the severity of this condition and the need for adequate interventions, if not already initiated. ${ }^{6}$

\section{How to reveal and measure organ dysfunction}

Organ dysfunction can be represented by an increase in the sequential [sepsis-related] organ failure assessment (SOFA) score, an objective and simple score, based on laboratory (platelet count, total bilirubin, serum creatinine) and clinical (blood pressure, Glascow coma scale, $\mathrm{PaO}_{2} / \mathrm{FiO}_{2}$ ratio) parameters combined with the need of therapeutic supports (vasopressors, mechanical ventilation). The baseline 
SOFA score can be assumed to be zero in patients without known preexisting organ failure, while a score of 2 points or more is associated with an in-hospital mortality greater than $10 \%$.

In out-of-ICU settings, adult individuals with suspected infection at high risk to have organ dysfunction and consequently sepsis, can be rapidly identified in early stage if they have at least 2 of the following clinical criteria constituting together a new bedside clinical score named quickSOFA (qSOFA): i) respiratory rate $\geq 22 / \mathrm{min}$; ii) altered mentation (Glasgow coma scale $\leq 15$ ); iii) systolic blood pressure $\leq 100 \mathrm{mmHg}$.

Outside the ICU, qSOFA had a high predictive validity for in-hospital mortality in a large retrospective cohort. This new measure does not require laboratory tests and is a simple bedside, quick and repeatable tool to identify adult patients with suspected infection at high risk to have poor outcomes. A $q S O F A \geq 2$ it should lead clinicians to further investigate for organ dysfunction (using SOFA), to start or escalate therapy, to increase the frequency of monitoring and to research for a possible infection, if not previously diagnosed. ${ }^{6}$

\section{Septic shock}

Septic shock is defined if hypotension not-responsive to fluid administration, requiring the vasopressor use to maintain the target mean arterial pressure (MAP), and high lactate level are present in the absence of hypovolemia. This clinical feature needs early diagnosis and a prompt treatment because septic shock is associated with mortality rates of $54 \%$.

The new clinical criteria for septic shock include: i) presence of sepsis; ii) fluid-unresponsive hypotension (blood pressure $\leq 90 \mathrm{mmHg}$ or $\mathrm{MAP} \leq 65 \mathrm{mmHg}$ ); iii) serum lactate $\geq 2 \mathrm{mmol} / \mathrm{L}(\geq 18 \mathrm{mg} / \mathrm{dL})$; iv) need for vasopressors to maintain MAP $\geq 65 \mathrm{mmHg}$. ${ }^{7}$

The proposal of these new diagnostic criteria needs further validation in clinical practice with dedicated prospective studies.

\section{Treatment}

When hemodynamic instability occurs, the patients need to be managed in monitored beds or in intensive care units.
The bases of the treatment of sepsis and septic shock are: i) antibiotics; ii) fluids (crystalloids or colloids); iii) vasopressors; iv) supportive tools (i.e., noninvasive mechanical ventilation, central venous catheter, noninvasive continue monitoring).

For which we refer to the dedicated chapters of this review.

\section{Conclusions}

In conclusion in recent years a better performance in diagnostic tools, the awareness campaigns, and the ageing of population increased the prevalence and the incidence of septic disease, making limited resources such as intensive care beds. This phenomenon has led to an increase in the number of cases treated in wards of internal or emergency medicine. Thus the training of modern internists cannot ignore the knowledge of the intensive use of antibiotics, fluids and vasopressors and the appropriate indication of further supportive care and diagnostics (i.e., noninvasive mechanical ventilation, bedside ultrasound).

\section{References}

1. Mazzone A, Dentali F, La Regina M, et al. Clinical features, short-term mortality, and prognostic risk factors of septic patients admitted to internal medicine units: results of an Italian Multicenter Prospective Study. Medicine (Baltimore) 2016;95:e2124.

2. Shankar-Hari M, Bertolini G, Brunkhorst FM, et al. Judging quality of current septic shock definitions and criteria. Crit Care 2015;19:445.

3. Bone RC, Balk RA, Cerra FB, et al. Definitions for sepsis and organ failure and guidelines for the use of innovative therapies in sepsis. Chest 1992;101:1644-55.

4. Levy MM, Fink MP, Marshall JC, et al. 2001 SCCM/ESICM/ACCP/ATS/SIS International Sepsis Definitions Conference. Crit Care Med 2003;31:1250-6.

5. Rhee C, Kadri SS, Danner RL, et al. Diagnosing sepsis is subjective and highly variable. Crit Care 2016;20:89.

6. Seymour CW, Liu VX, Iwashyna TJ, et al. Assessment of Clinical Criteria for Sepsis. For the Third International Consensus Definitions for Sepsis and Septic Shock (Sepsis-3) JAMA 2016;315:762-774.

7. Seymour CW, Rosengart MR. Septic shock advances in diagnosis and treatment. JAMA 2015;314:708-717. 